\title{
Relation between Thermal Conductivity and Viscosity for Nonpolar Gases. II. Rotational Relaxation of Polyatomic Molecules
}

\author{
Clevelaxd O'Neal, Jr., and Richard S. Brokaw \\ Lewis Research Center, National Aeronautics and Space Administration, Cleceland, Ohio \\ 15842 \\ (Received 7 June, 1963) \\ The dimensionless ratio $f=\lambda . M / \eta C$, relating the thermal conductivit $y$, molecular weight, viscosity, \\ and constant volume molar heat capacity has been determined for several nonpolar polyatomic gases \\ in the neighborhood of room temperature $\left(270^{\circ}-295^{\circ} \mathrm{K}\right.$ ). The experimental method, due to Eckert. \\ and Irvine, provides a direct determination of $f$ by measurement of the subsonic temperature recovery \\ factor. A recent theory of Mason and Monchick has been used to calculate collision numbers for \\ rotational relaxation from the experimental data as follows: $\mathrm{CH}_{4}, 9.4 ; \mathrm{CF}_{4}, 3.0 ; \mathrm{SF}_{6}, 2.5 ; \mathrm{C}_{2} \mathrm{H}_{4}, 2.4$; \\ $\mathrm{C}_{2} \mathrm{H}_{6}, 4.0 ; \mathrm{O}_{2}, 12 ; \mathrm{N}_{2}, 7.3 ; \mathrm{CO}_{2}, 2.4$; and $\mathrm{C}_{2} \mathrm{H}_{2}, 1.8$. Collision numbers for the near-spherical molecules \\ were in close accord with a classical theory for rough sphere molecules with attractive forces; ethyl- \\ ene, which deviates appreciably from spherical symmetry, exhibited a smaller collision number. The \\ data on linear molecules were in qualitative agreement with a quantum treatment. In general, col- \\ lision numbers for rotationai reiaxationi are determined by the following factors: (1) The molecular \\ mass distribution, (2) the strength of the intermolecular attractive forces, and (3) ihe wiscicular \\ asymmetry. \\ AUTHOR
}

\section{INTRODUCTION}

$I^{\mathrm{N}}$ $\mathrm{N}$ an earlier paper ${ }^{1}$ we reported experimental values of the dimensionless ratio

$$
f=\lambda M / \eta C_{v},
$$

which relates the thermal conductivity, molecular weight, viscosity, and constant volume molar heat capacity. The experimental method, due to Eckert and Irvine, ${ }^{2}$ provides a direct determination of $f$ by measurement of the subsonic temperature recovery factor. Helium, argon, nitrogen, oxygen, and hydrogen were studied in the range $90^{\circ}-290^{\circ} \mathrm{K}$.

The data on helium and argon were in close accord with the rigorous Chapman-Enskog kinetic theory for monatomic gases, ${ }^{3}$ for which $f$ is very slightly larger than 2.5 . The results for nitrogen,

${ }^{1}$ C. O'Neal, Jr., and R. S. Brokaw, Phys. Fluids 5, 567 (1962).

2 E. R. G. Eckert and T. F. Irvine, Jr., J. Appl. Mech. 24, $25(1957)$.

${ }^{3} \mathrm{~S}$. Chapman and T. G. Cowling, The Mathematical Theory of Non-Uniform Gases (Cambridge Liniversity Press, New York, 1952), p. 235. oxygen, and hydrogen, as well as Novotny and Irvine's data on carbon dioxide, ${ }^{4}$ were analyzed in terms of Mason and Monchick's ${ }^{5}$ theory for polyatomic gases, which relates deviations from the modified Eucken approximation to the relaxation times of internal energy states. Collision numbers for rotational relaxation for nitrogen, oxygen, and carbon dioxide were found to be nearly independent of temperature. The results confirmed the fact that the interchange of translational and rotational energy is unusually difficult for hydrogen.

Mason and Monchick's ${ }^{5}$ theory for nonpolar polyatomic gases may be written

$$
\begin{aligned}
f C_{r}=\frac{5}{2} C_{\mathrm{rtrans}} & +\frac{\rho D}{\eta} C_{\mathrm{sint}} \\
& -\frac{1}{2}\left(\frac{5}{2}-\frac{\rho D}{\eta}\right)^{2} \sum_{k} \frac{\eta}{p \tau_{k}} C_{\mathrm{v} k} .
\end{aligned}
$$

${ }_{4}^{4}$ L. Novotny and T. F. Irvine, Jr., J. Heat Transfer 83, 125 (1961).

5 E. A. Máson and L. Monchick, J. Chem. Phys. 36, 1622 (1962) 
Here $\rho$ is the density and $D$ is the average coefficient for the diffusion of internal energy; for nonpolar gases it can be taken to be the self-diffusion coefficient. $C_{v_{\text {trans }}}$ and $C_{v_{\text {int }}}$ are the translational and internal contributions to the heat capacity $C_{v} ; \tau_{k}$ is the relaxation time for the $k$ th internal energy mode, and $C_{v k}$ is the heat capacity of that mode, while $p$ is the pressure.

The first two terms in Eq. (2) are simply the modified Eucken approximation, developed by Chapman and Cowling, ${ }^{6}$ Schafer, ${ }^{7}$ and Hirschfelder. ${ }^{8}$ The third term is important only for small relaxation times. In small, rigid polyatomic molecules these are the rotational relaxation times; in flexible molecules, the vibrational relaxation times may also be small. (The only nonrigid molecule considered in this paper is ethane.)

It is further convenient to express the relaxation times in terms of a collision number

$$
Z_{k}=\frac{\tau_{k}}{\tau_{\mathrm{coll}}}=\frac{4}{\pi} \frac{p \tau_{k}}{\eta}
$$

Consequently, for nonpolar gases

$$
f=f_{\text {mod. Eucken }}-\frac{2}{\pi C_{n}^{\prime}}\left(\frac{5}{2}-\frac{\rho D}{\eta}\right)^{2} \sum_{k} \frac{C_{n k}^{r}}{Z_{k}} .
$$

This paper reports $f$ values in the vicinity of room temperature for several nonpolar polyatomic molecules, again determined using Eckert and Irvine's ${ }^{2}$ experimental technique. Nonlinear molecules, possessing three rotational degrees of freedom, were methanc, tetrafluoromethane, sulfur hexafluoride, ethane, and ethylene. Linear molecules, with two rotational degrees of freedom, included oxygen, nitrogen, carbon dioxide, and acetylene (some preliminary results on methane and the linear molecules have already been reported elsewhere ${ }^{9}$ ). Collision numbers for rotational relaxation have been computed from Eq. (4) and interpreted in terms of the existing theories of rotational relaxation. ${ }^{10,11}$ This provides considerable insight as to the molecular parameters which determine rotational relaxation rates.

\section{EXPERIMENTAL}

We have previously described the apparatus and experimental procedure for measuring recovery fac-

${ }^{6}$ Reference 3, p. 238.

7 K. Schäfer, Z. Physik. Chem. B53, 149 (1943)

8 J. O. Hirschfelder, J. Chem. Phys. 26, 282 (1957)

9 R. S. Brokaw and C. O'Neal, Jr., Ninth Symposium (International) on Combustion (Academic Press Inc., New York, 1963), p. 725.

${ }_{10}$ N. F. Sather and J. S. Dahler, J. Chem. Phys. 37, 1947 (1962)

${ }^{11}$ R. Brout, J. Chem. Phys. 22, 1189 (1954). tors, ${ }^{1}$ and the method has been discussed at length by Irvine. ${ }^{12}$ Consequently, the technique will merely be outlined here. The method is based on the relation describing the adiabatic temperature attained by a flat plate in a subsonic gas stream. This recovery temperature $T_{\mathrm{r}}$ is conveniently described by a recovery factor

$$
r \equiv\left(T_{\mathrm{r}}-T_{\mathrm{s}}\right) /\left(T_{\mathrm{t}}-T_{\mathrm{s}}\right),
$$

where $T_{\mathbf{s}}$ is the static temperature of the stream at a sufficient distance from the plate and $T_{\mathrm{t}}$ is the total temperature (the temperature measured at a stagnation point). Pohlhausen, ${ }^{13}$ by integrating the laminar boundary layer equations, found that the recovery factor is a function of Prandtl number $C_{p} \eta / M \lambda$ alone. Spence $^{14}$ has shown that for Prandtl numbers near unity

$$
r \cong\left(C_{p} \eta / M \lambda\right)^{\frac{1}{2}}=\left(C_{p} / f C_{v}\right)^{\frac{1}{2}}
$$

Indeed Eq. (6) reproduces numerical calculations to better than sixteen parts in ten thousand for Prandtl numbers between $\frac{2}{3}$ and 1 . $^{9}$

To measure the recovery factor, the gas is expanded through a convergent nozzle; the pressure ratio is such that the issuing gas remains slightly subsonic. A butt-welded differential thermocouple is suspended along the nozzle axis with one junction in the low velocity region upstream of the nozzle sensing the total temperature and the other junction just downstream of the nozzle exit assuming the flat plate recovery temperature. The static temperature is not measured; rather, it is computed from the pressure ratio across the nozzle assuming iscntropic flow.

The dimensionless entropy of a gas may be written ${ }^{15}$

$$
\frac{S}{R}=\frac{S^{0}}{R}-\ln p-\frac{p}{R} \frac{d B}{d T}+\cdots .
$$

Here $S$ is the entropy, $S^{0}$ is the entropy of the ideal gas at one atmosphere, $R$ is the gas constant, $p$ is the pressure, $B$ is the second virial coefficient, and $T$ is the absolute temperature. Consequently, for isentropic flow,

$$
\frac{S_{T_{\mathrm{a}}}^{0}}{R}=\frac{S_{T_{\mathrm{t}}}^{0}}{R}-\ln \frac{p_{\mathrm{t}}}{p_{\mathrm{s}}}-\frac{p_{\mathrm{t}}}{R}\left(\frac{d B}{d T}\right)_{r=T_{\mathrm{t}}}+\frac{p_{\mathrm{a}}}{R}\left(\frac{d B}{d T}\right)_{T=T_{\mathrm{s}}} .
$$

The last two terms in Eq. (8) are a small correction

${ }_{12}$ T. F. Irvine, Jr., Ph.D. Thesis, University of Minnesota, 1956.

${ }_{13}$ E. Pohlhausen, Z. Angew. Math. u. Mech. 1, 115 (1921).

14 D. A. Spence, J. Aerospace Sci. 27, 878 (1960).

is J. O. Hirschfelder, C. F. Curtiss, and R. B. IBird, Molecular Theory of Gases and Liquids (John Wiley \& Sons, Inc., New York, 1954) Eg. 3. B-3, p. 231. 
due to deviations from the ideal gas law. To compute the stream temperature an approximate value was obtained from Eq. (8) using tabulated standard entropies and the experimental pressure ratio. The derivatives of the virial coefficients were then calculated assuming a Lennard-Jones (12-6) potential together with appropriate force constants. ${ }^{16}$ Equation (8) was then used to calculate the final value of

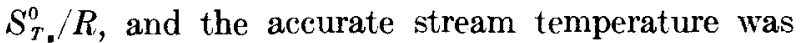
obtained by interpolation in the table of entropies. Recovery factors and values of $f$ could then be calculated from Eqs. (5) and (6). Eutropies and heat capacities for oxygen, nitrogen, and ethane were taken from National Bureau of Standards compilations, ${ }^{17,18}$ while values for the remaining species were calculated by the methods discussed by McBride, Heimel, Ehlers, and Gordon, ${ }^{19}$ using their spectroscopic constants and computer program.

The present measurements were all carried out at room temperature; hence, temperature control was no problem. Furthermore, it was possible to work with fairly high flow rates so that the pressure ratios and differential thermocouple emf's could be read with greater accuracy. As a consequence, the present measurements show considerably less scatter than oùr previous data. ${ }^{1}$

In order to detect instrumental errors, 83 check determinations of the recovery factor of argon were interspersed throughout the course of this work. These values correspond to a temperature of about $275^{\circ} \mathrm{K}$ and yield a mean value of $f=2.5028$ with a standard error of 0.0015 . (The standard deviation of each datum was 0.0135.) This is in excellent agreement with the theoretical value of $f=2.5023$, and may be regarded as a calibration of the apparatus.

\section{RESULTS AND DISCUSSION}

Experimental $f$ values are presented in Table I together with the corresponding temperatures. Also shown are values of $Z_{\text {rot }}^{-1}$, the reciprocal of the collision number for rotational relaxation, calculated from Eq. (4). In calculating $Z_{\text {rot }}^{-1}$ the relation $\rho D / \eta=$ $\left(\frac{6}{5}\right) A^{*}$ has been invoked. ${ }^{20} A^{*}$ is a very slowly varying function of temperature; values were obtained assuming either the exponential -6 or Lennard-Jones (12-6) potential. Also shown in Table I are mean

16 Reference 15, Tables I-A and I-B, pp. 1100-1115. $17 \mathrm{~J}$. Hilsenrath, et al., Tables of Thermal Properties of Gases, Natl. Bur. Std. Circ. 564, (1955).

${ }^{18}$ F. D. Rossini, et al., Selected Talues of Properties of Hydrocarbons, Natl. Bur. Std. Cire. 461 (1947).

${ }_{19}$ B. J. McBride, S. Heimel, J. G. Ehlers, and S. Gordon, NASA Special publication SP-3001, 1963.

20 Reference 15, Eq. 8.2-18, p. 540. temperatures $\tilde{T}$; reciprocal collision numbers $\overline{Z_{\text {rot }}^{-1}}$ standard deviations $\bar{\sigma}$; and standard errors of the mean $\bar{e}$.

For all of the molecules except ethane a thousand or more collisions are required for vibrational relaxation; hence, vibrational effects could be ignored in Eq. (4). In ethane the mode corresponding to hindered rotation about the carbon-carbon bond has a relaxation time of $1.24 \times 10^{-9} \mathrm{sec}$, or $17 \mathrm{col}-$ lisions, at $296.4^{\circ} \mathrm{K}^{21}$ Assuming that a $3 \mathrm{kcal}$ barrier hinders internal rotation, the tabulations of Pitzer and $\mathrm{Gwinn}^{22}$ give $C / R=0.999$ for this mode at $286^{\circ} \mathrm{K}$. The rotational collision numbers for ethane have been calculated assuming this heat capacity and collision number for internal rotation; if the internal rotation were ignored, ethane $Z_{\text {rot }}^{-1}$ values would all be larger by 0.040 .

Collision numbers determined from recovery factor, or $f$, are compared with other measurements in Table II. The present values are generally in accord with those obtained by other techniques, within the admittedly rather large uncertainties associated with such determinations. The results for oxygen seem to fall into two groups: collision numbers in the ranges of $2-6$ and $12-30$. Our value falls in the latter category. The only other serious disagreement is in the case of carbon dioxide, where acoustical measurements indicate that sixteen collicions are rernuired for relaxation. We are inclined to believe the acoustic result is in error-we can think of no property of the carbon dioxide molecule which would cause such long relaxation times.

In order to compare our collision numbers with theoretical calculations, it is desirable to divide the molecules into two groups, linear and nonlinear, possessing two and three rotational degrees of freedom, respectively. We chose to discuss the nonlinear molecules first, since a classical theory for nonlinear molecules with attractive intermolecular forces (rough spheres and spherocylinders surrounded by square wells) has recently been developed..$^{10}$

\section{Nonlinear Molecules}

Sather and Dahler ${ }^{10}$ obtained the following expression for the rotational relaxation time of a rough sphere with a spherically symmetric, square, attractive well:

$$
\tau_{\text {rot }}^{-1}=\frac{16}{3} n \sigma^{2} \frac{\left(4 I / m \sigma^{2}\right)}{\left[1+\left(4 I / m \sigma^{2}\right)\right]^{2}}\left(\frac{\pi k T}{m}\right)^{\frac{1}{2}} g(\sigma) .
$$

${ }^{21}$ I. M. Valley and S. Legvold, J. Chem. Phys. 33, 627 $(1960)$.

${ }^{22}$ K. S. Pitzer and W. D. Gwinn, J. Chem. Phys. 10, $428(1942)$. 
TABLE I. Experimental Results

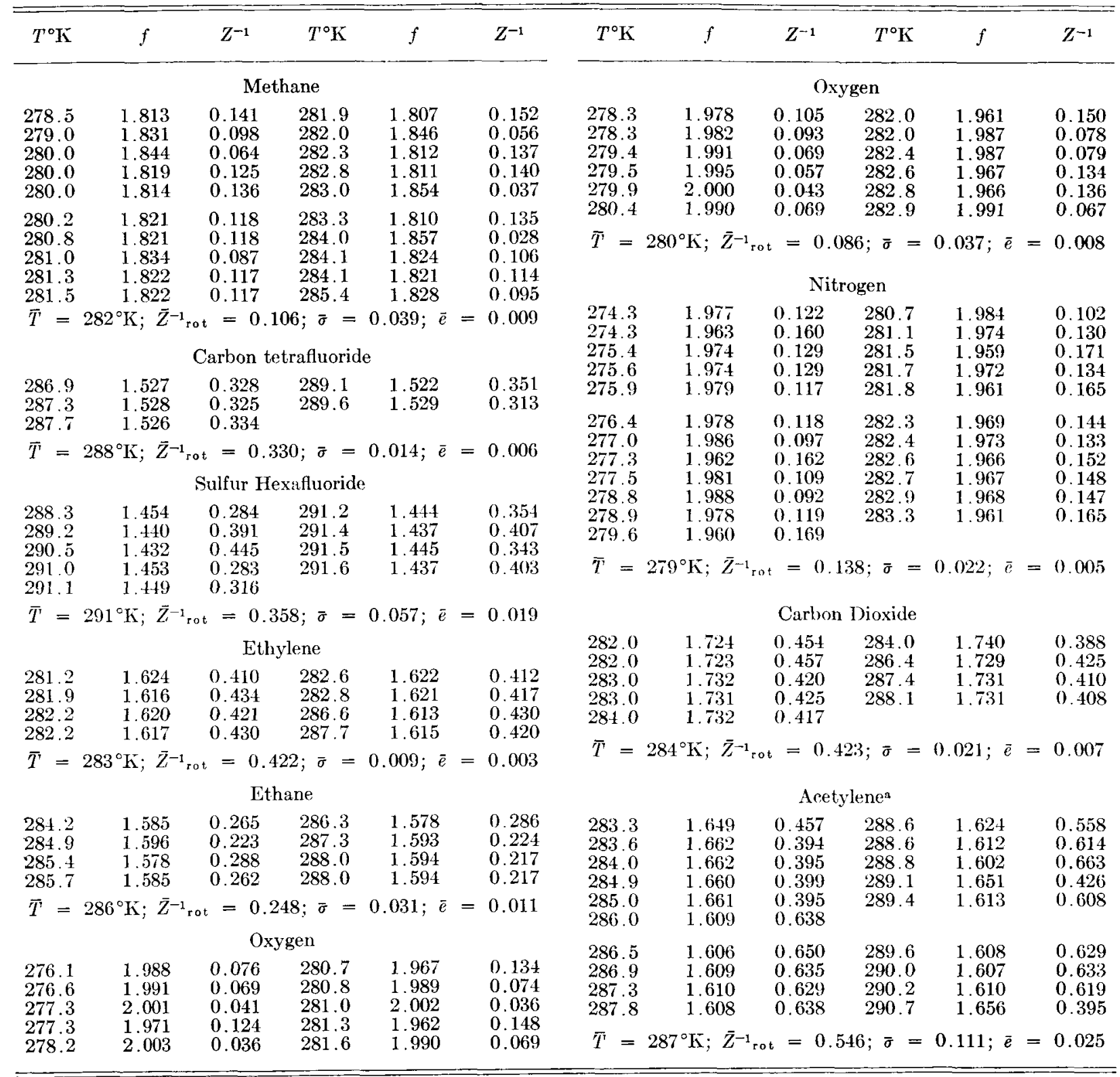

The data on acetylene fall into two distinct groups. For the most recent data $\bar{Z}^{-1}$ rot $=0.626$, whereas, for the earlier results $\vec{Z}^{-1}$ rot $=0.409$ This is the cause of the high standard deviation $\tilde{\sigma}$. We have no valid reason to reject the earlier data although we have been unable to reproduce them.

Here $n$ is the number of molecules per $\mathrm{cm}^{3}, I$ is the moment of inertia, $m$ is the molecular mass, $k$ is the Boltzmann constant, $\sigma$ is the diameter of the rough sphere core, while $g(\sigma)$ is the value of the radial distribution function at $\sigma$. In the low density limit, ${ }^{23}$ $g(\sigma)=\exp (\epsilon / k T)$, where $\epsilon$ is the depth of the well at $\sigma$.

Two features of Eq. (9) are noteworthy. First, since the quantity $4 I / m \sigma^{2}$ is generally less than 0.2 ,

${ }^{23}$ Reference 15, Eq. 4.9-1, p. 321 , the relaxation time is only weakly dependent on $\sigma$, the position of repulsive core. Secondly, since there is no rotational energy transfer accompanying the velocity impulse at the outer edge of the potential well, the only contribution to the rotational relaxation stems from the impulse at the core. Thus, the width of the well is not important; in fact, Eq. (9) should apply to a Sutherland potential with a rough core - that is, a potential with an inverse sixth power attractive portion to account for van der Waals forces. 
TABLE II. Comparison of Rotational Collision Numbers

\begin{tabular}{|c|c|c|c|c|c|}
\hline \multirow[t]{2}{*}{ Gas } & \multicolumn{5}{|c|}{ Experimental method } \\
\hline & $\begin{array}{l}\text { Recovery } \\
\text { factor }\end{array}$ & $\begin{array}{c}\text { Acous } \\
\text { tical }\end{array}$ & $\begin{array}{c}\text { bLow pressure } \\
\text { thermal } \\
\text { conductivity }\end{array}$ & $\begin{array}{l}\text { Shock } \\
\text { thick- } \\
\text { ness }\end{array}$ & $\begin{array}{c}\text { Impact } \\
\text { tube }\end{array}$ \\
\hline $\mathrm{N}_{2}$ & 7 & $5.3,6,4-6$ & & $5.5^{\mathrm{c}}$ & $\begin{array}{l}<7 \mathrm{~d}^{\mathrm{d}} \\
<1 \mathrm{e}^{\mathrm{e}}\end{array}$ \\
\hline $\mathrm{O}_{2}$ & 12 & $\begin{array}{c}2-4,4.1,12 \\
14,12-30\end{array}$ & 20 & $7^{\mathrm{s}}$ & \\
\hline $\mathrm{H}_{2}$ & Largeg & $240-360$ & 300 & $>150^{\prime}$ & $\begin{array}{l}160,{ }^{d} \\
310^{e}\end{array}$ \\
\hline $\begin{array}{l}\mathrm{CO}_{2} \\
\mathrm{CH}_{4}\end{array}$ & $\begin{array}{l}2.4 \\
9\end{array}$ & $\begin{array}{c}16 \\
14-17\end{array}$ & & $1-2^{\circ}$ & \\
\hline
\end{tabular}

- Cited by T. L. Cottrell and J. C. McCoubrey, Molecular Energy Transfer in Gases (Butterworths Scientific Publications Ltd., London 1961) Ch. is.

b F. G. Waelbroeck and P. Zukerbrodt, J. Chem. Phys. 28, 524 (1958). - W. H. Andersen and D. F. Hornig. Mol. Phys. 2, 49 (1959)

d P. W. Huber and A. Kantrowitz. J. Chem. Phys. 15, 275 (1947).

- W. Griffith, J. Appl. Phys. 21, 1319 (1950).

I E. F. Greene and D. F. Hornig. J. Chem. Phys. 21, 617 (1953).

- Reference 1.

The coefficient of viscosity is

$$
\eta=\left(5 / 16 \sigma_{\eta}^{2}\right)(m k T / \pi)^{\frac{2}{3}},
$$

where $\sigma_{\eta}^{2}$ is the square of the viscosity collision diameter $\left[\equiv \sigma^{2} \Omega^{(2,2) *}\right.$ in the notation of reference 15 , page 527, Eq. 8.2-10]. Thus, from Eqs. (3) and (9) together with the viscosity formula and the perfect gas law,

$$
\begin{aligned}
Z_{\mathrm{rot}}^{-1} & =\frac{5 \pi}{12} \frac{\left(4 I / m \sigma_{\eta}^{2}\right)}{\left[1+\left(4 I / m \sigma^{2}\right)\right]^{2}} \exp \left(\frac{\epsilon}{k T}\right) \\
& \cong \frac{5 \pi}{12}\left(\frac{4 I}{m \sigma_{\eta}^{2}}\right) \exp \left(\frac{\epsilon}{k T}\right) .
\end{aligned}
$$

We might expect that the rough sphere model with an attractive potential well would apply to nonlinear molecules which are approximately spherical.

The experimental results on nonlinear molecules are analyzed in Table III. Viscosity collision diam-
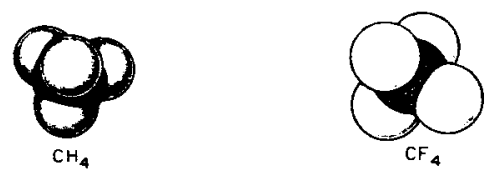

Fig. 1. Configurations of nonlinear molecules.
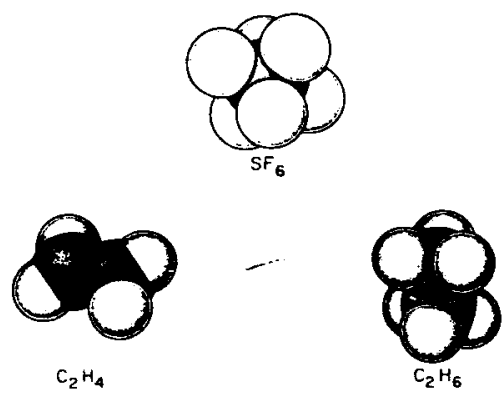

eters were calculated assuming a Lennard-Jones (12-6) potential for all molecules except methane; for methane the exponential -6 potential was used. Methane, carbon tetrafluoride, and sulfur hexafluoride are approximately spherical as shown in Fig. 1. Indeed, $Z_{\text {rot }}^{-1}$ values for these molecules calculated from both Eqs. (10a) and (10b) are in satisfactory agreement with experiment. [The approximation $(10 \mathrm{~b})$ is actually in closer accord with experiment, but it is not clear that this is significant.] The mass distribution parameter $4 I / m \sigma_{\eta}^{2}$ varies more than threefold between $\mathrm{CH}_{4}$ and $\mathrm{CF}_{4}$; thus, the large collision number for methane is undoubtedly a direct consequence of the molecule's small moment of inertia. Note, too, that the effect of attractive forces [exp $(\epsilon / k T)]$ is appreciable and roughly doubles the transition probabilities.

The $Z_{\text {rot }}^{-1}$ values for ethylene and ethane were calculated using average moments of inertia. Values for ethane are also in close accord with experiment, but in the case of ethylene the agreement is not so

\begin{tabular}{|c|c|c|c|c|c|}
\hline & Methane & $\begin{array}{c}\text { Carbon } \\
\text { tetrafluoride }\end{array}$ & $\begin{array}{c}\text { Sulfur } \\
\text { hexafluoride }\end{array}$ & Ethylene & Ethane \\
\hline Type of potential & $\exp -6^{\mathrm{a}}$ & L-J $12-6$ & L-J $12-6$ & \multirow{5}{*}{\multicolumn{2}{|c|}{$\begin{array}{cc}\mathrm{I}-\mathrm{J} 12-6 & \mathrm{~L}-\mathrm{J} 12-6 \\
224.7 & 215.7 \\
4.163 & 4.443 \\
\cdots & \cdots \\
5.75 & 11.03 \\
28.09 & 42.28\left(^{2}\right) \\
33.84 & \end{array}$}} \\
\hline $\int \epsilon / k,{ }^{\circ} \mathbf{K}$ & 152.8 & 134.0 & 222.1 & & \\
\hline Force constants $\left\{\sigma\right.$ or $r_{\alpha}, \AA$ & 4.206 & 4.662 & 5.128 & & \\
\hline $\begin{array}{l}\text { Moments of inertia, } \\
\mathrm{gm} \mathrm{cm}^{2} \times 10^{40}\end{array}$ & 5.341 & \multirow[t]{2}{*}{147.3} & \multirow[t]{2}{*}{307.1} & & \\
\hline $4 \mathrm{I} / m \sigma^{2}$ & 00476 & & & & \\
\hline $\exp (\epsilon / k T)$ & $\begin{array}{l}0.072 \\
1.72\end{array}$ & 1.59 & 2.14 & $\begin{array}{l}0.018 \pm \\
2.21\end{array}$ & $\begin{array}{l}0.0929 \\
2.13\end{array}$ \\
\hline$Z^{-1}$ rot $[\mathrm{Eq} \cdot(10 \mathrm{a})]$ & 0.098 & 0.249 & 0.297 & 0.195 & 0.217 \\
\hline$Z^{-1} \operatorname{rot}[$ Eq. $(10 \mathrm{~b})]$ & 0.107 & 0.335 & 0.384 & 0.227 & 0.259 \\
\hline$Z^{-1}$ rot Experiment & 0.106 & 0.330 & 0.358 & 0.422 & 0.248 \\
\hline Standard error of the mean & 0.009 & 0.006 & 0.019 & 0.003 & 0.011 \\
\hline
\end{tabular}
good; the predicted $Z_{\mathrm{rot}}^{-1}$ are roughly half of experi-

TABLE III. Comparison of Theory and Experiment for Nonlinear Molecules

- Reference 29 


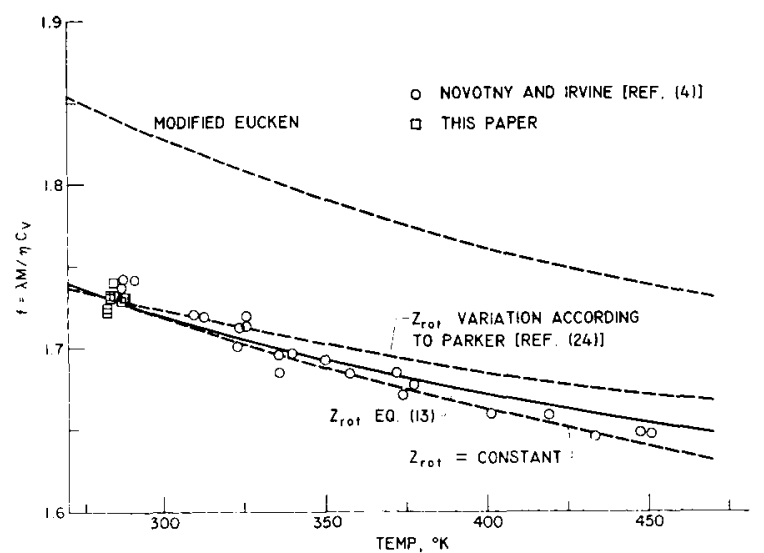

FIG. 2. Experimental and theoretical values of $f$ for carbon dioxide.

ment. From Fig. 1 it is apparent that the ethane molecule is approximately spherical, whereas the ethylene structure is definitely less compact and symmetric.

It appears, then, that the classical kinetic theory for a rough spherical molecule with an attractive well [Eq. (10a)] may provide a method for calculating a lower limit to the collision probability for rotational relaxation, $Z_{\text {rot }}^{-1}$, for nonlinear molecules. Molecules such as $\mathrm{CH}_{4}, \mathrm{CF}_{4}, \mathrm{SF}_{6}$, and $\mathrm{C}_{2} \mathrm{H}_{8}$ are reasonably represented by this model. Less symmetric molecules such as $\mathrm{C}_{2} \mathrm{H}_{4}$ exhibit larger transition probabilities, or shorter relaxation times, perhaps due to longer range asymmetries in the intermolecular force field.

\section{Linear Molecules}

Brout $^{11}$ has developed a quantum mechanical treatment for the rotational relaxation of homonuclear diatomic molecules. He idealizes one of the molecules as a mass point and expresses the intermolecular potential $\Phi$ in the form

$$
\Phi(r)=A e^{-\beta r}\left[1+\psi P_{2}(\cos \theta)\right] .
$$

Here $P_{2}(\cos \theta) \equiv \frac{1}{2}\left(3 \cos ^{2} \theta-1\right)$ is the Legendre polynomial of order 2 , and $\theta$ is the angle between the line of centers of the colliding molecules and the figure axis of one of the molecules. $\psi$ is a parameter describing the deviation of the potential from spherical symmetry. For such a potential, the transition probability may be written

$$
Z_{\mathrm{rot}}^{-1}=\frac{25}{28} \psi^{2}\left(4 I / m \sigma_{\eta}^{2}\right)
$$

Although Brout developed Eq. (12) for homonuclear diatomic molecules, it should apply as well to symmetrical linear polyatomic molecules such as carbon dioxide, acetylene, etc. Note that the mass distri- bution parameter $4 I / m \sigma_{\eta}^{2}$ is again relevant, as in the classical rough sphere case.

The potential function given in Eq. (11) is entirely repulsive, whereas real molecules have long range attractive forces. It would seem reasonable to account for the attractive forces by a multiplicative factor $\exp (\epsilon / k T)$ by analogy with Eq. (10):

$$
Z_{\mathrm{rot}}^{-1}=\frac{25}{28} \psi^{2}\left(\frac{4 I}{m \sigma_{\eta}^{2}}\right) \exp \left(\frac{\epsilon}{k T}\right) .
$$

Equation (13) indicates some variation of collision number with temperature. Assuming that $\psi$ is independent of temperature-and, as we shall see, Brout's treatment indicates that it is-this variation can be calculated. Thus, over the range of experimental measurement-roughly $100^{\circ}$ to $300^{\circ} \mathrm{K}^{-1}$ Eq. (13) indicates that $Z_{\text {rot }}^{-1}$ for nitrogen should decrease by $25 \%$ and $Z_{\text {ro }}^{-1}$ for oxygen should decrease by $35 \%$. This temperature variation represents the somewhat scattered data ${ }^{1}$ on these molecules about as well as a temperature independent collision number. The experimental data on carbon dioxide ${ }^{4} \mathrm{ex}-$ tend from about $280^{\circ}$ to $460^{\circ} \mathrm{K}$ and are more precise. Over this range $\mathrm{Eq}$. (13) indicates a $9 \%$ decrease in $Z_{\text {rot }}^{-1}$. In Fig. 2, the carbon dioxide data are shown together with calculated curves assuming: First, a temperature independent collision number, next, the temperature variation of Eq. (13), and finally, the temperature variation of Parker's ${ }^{24}$ classical two-dimensional treatment for diatomic molecules. The data are best represented by a constant collision number, although Eq. (13) is also reasonably satisfactory. Parker's temperature dependence would appear to be ruled out. It might be noted that Sather and Dahler ${ }^{10}$ calculated the effect of temperature on the relaxation times for spherocylinders and found a somewhat less pronounced temperature variation than in the rough sphere case. Thus, it would seem that if there is a long range asymmetry in the attractive potential there may be less effect of temperature on the relaxation time.

The experimental results on linear molecules are analyzed in Table IV. Experimental values of $\psi$ have been computed from Eq. (13); it is seen that $\psi$ is of the order of unity, varying from 0.7 for oxygen to 1.7 for acetylene. Brout ${ }^{25}$ has shown how $\psi$ for diatomic molecules can be calculated assuming that the total potential is made up of the sum of atom-atom interactions,

$$
\Phi(r)=\sum \Phi_{i j},
$$

${ }^{24}$ J. G. Parker, Phys. Fluids 2, 449 (1959).

${ }_{25}$ R. Brout, J. Chem. Phys. 22, 934 (1954). 
Table IV. Comparison of Theory and Experiment for Linear Molecules

\begin{tabular}{|c|c|c|c|c|}
\hline & Oxygen & Xitrogen & $\begin{array}{l}\text { Carbon } \\
\text { dioxide }\end{array}$ & Acetylene \\
\hline Type of potential & \multirow{5}{*}{$\begin{array}{c}{ }^{a} \exp -6 \\
132 \\
3.726 \\
17.0 \\
19.3\end{array}$} & \multirow{5}{*}{$\begin{array}{c}{ }^{b} \exp -6 \\
101.2 \\
4.011 \\
17.0 \\
14.01\end{array}$} & \multirow{5}{*}{$\begin{array}{c}c \mathrm{~L}-\mathrm{J} J 2-6 \\
195.2 \\
3.941 \\
71.47\end{array}$} & \multirow{4}{*}{$\begin{array}{c}{ }^{c} \mathrm{~L}-\mathrm{J} \\
231.8 \\
4.033 \\
\ldots\end{array}$} \\
\hline$\left(\epsilon / k,{ }^{\circ} \mathbf{K}_{0}\right.$ & & & & \\
\hline Force constants $\left\{\sigma\right.$ or $r_{\mathrm{m}}, \AA$ & & & & \\
\hline Noment of inertia $\quad \alpha$ & & & & \\
\hline $\begin{array}{l}\text { Moment of inertia, } \\
\text { gm } \mathrm{cm}^{2} \times 10^{40} \\
\text { Data analysis }\end{array}$ & & & & 23.65 \\
\hline $4 \mathrm{I} / m \sigma^{2} \eta$ & 0.111 & 0.086 & 0.189 & 0.094 \\
\hline $\exp (\epsilon / k T)$ & 1.60 & 1.44 & 1.99 & 2.24 \\
\hline$\psi$ expt. [Eq. (13)] & 0.74 & 1.11 & 1.12 & 1.71 \\
\hline $\int \beta=2.5$ & 0.55 & 0.48 & 1.16 & 1.48 \\
\hline$\psi$ calc $\{\beta=4.5$ & 1.10 & 1.01 & 1.63 & 1.80 \\
\hline
\end{tabular}

J. T. Vanderslice, E. A. Mason, and W. G. Maisch, J. Chem. Phys. 31, 738 (1959).

c.R. A. Srehla, NASA Technical Report R-132, 1963.

where $\Phi_{i}$ is of the form

$$
\Phi_{i j}=A \exp \left(-\beta r_{i j}\right) .
$$

In this event

$$
\psi_{\text {di a torn ic }} \cong(\beta d)^{2} /\left[12+\frac{1}{2}(\beta d)^{2}\right],
$$

where $\beta$ is the index of the repulsive potential and $d$ is the internuclear distance. From Eq. (14) it is clear that $\psi$ lies in the range zero to two, which is in agreement with the experimental $\psi$ listed in Table IV.

Values of $\beta$ can be obtained from two sources. For the exponential repulsive potential between light atoms and molecules involving hydrogen ${ }^{28}$ and oxygen and nitrogen ${ }^{27} \beta$ lies in the range 2.5 to 3.5 . On the other hand, one can deduce values from force constants for the exponential -6 potential $\left(\beta=\alpha / r_{\mathrm{m}}\right)$; for light molecules $\beta$ so obtained lie in the range 3.5 to $4 . \bar{s}^{27,28}$

Accordingly, values of $\psi$ have been calculated from Eq. (14) assuming $\beta$ of 2.5 and 4.5 so as to cover the extreme range. For carbon dioxide or acetylene, $d$ in Eq. (14) was taken as the intramolecular oxygen-oxygen or hydrogen-hydrogen distance. In Table IV we see the experimental $\psi$ for oxygen and acetylene lie between the computed extremes while the experimental $\psi$ for nitrogen and carbon dioxide lie just outside the calculated range. In the case of the acetylene and carbon dioxide molecules one might expect the effective centers of repulsion to lie inside the hydrogen or oxygen atoms.

It is not difficult to extend Brout's treatment to molecules with multiple centers of repulsion (say one for each atom), and also to apply a correction for molecules which deviate more severely from

${ }^{26}$ J. T. Vanderslice, S. Weissman, E. A. Mason, and R. J. Fallon, Phys. Fluids 5, 155 (1962).

${ }^{27}$ K. S. Yun and E. A. Mason, Phys. Fluids 5, 380 (1962).

${ }^{28}$ Reference 15, Table 3.7-2, p. 181. spherical symmetry. However, because the uncertainty in the $\beta$ values remains, there is no substantial improvement over the results shown in Table IV.

It seems, then, that Brout's theory, corrected for attractive forces, describes the rotational relaxation of linear molecules, at least approximately. Accurate a priori calculation of relaxation times requires an accurate knowledge of the molecular phrenology, characterized by $\beta d$; such information is not generally available.

In a previous paper ${ }^{9}$ we noted an empirical correlation between the relaxation times of linear molecules and the hiviecular quadrunole moments. Accordingly, we attempted to express the potential in the form of Eq. (11) with the angularly dependent portion arising from the quadrupole-quadrupole interaction. First, it was necessary to average the potential over all orientations of one of the molecules. $^{29}$ Next, we had to drastically approximate a $\cos ^{4} \theta$ angular dependence as

$$
\cos ^{4} \theta \sim \overline{\cos ^{2} \theta} \cos ^{2} \theta=\frac{1}{3} \cos ^{2} \theta
$$

to obtain the admittedly very approximate expression

$$
\psi \sim \frac{1}{8} Q^{4} / r^{10} k T \Phi .
$$

Here $Q$ is the quadrupole moment. If we assume that transition occurs where $\Phi$ is of the order of $k T$ and $r$ is of the order of the viscosity collision diameter,

$$
\psi \sim \frac{1}{8} Q^{4} / \sigma_{\eta}^{10}(k T)^{2} .
$$

Consider carbon dioxide as an example: Buckingham $^{30}$ has recently determined the quadrupole moment as $-8.2 \times 10^{-26}$ esu. From Eq. (15) we obtain, for a temperature of $288^{\circ} \mathrm{K}, \psi \sim 0.1$. Thus,

${ }^{29}$ Reference 15 , pp. 27-28, 985.

${ }^{30}$ A. D. Buckingham, Abstracts of Papers, 144th National Meeting, American Chemical Society, Los Angeles, California, March 31-April 5, 1963. 
we suspect that the quadrupole interaction is not important in inducing translation-rotation transitions; rather; the correlation previously observed arises because the deviations from spherical symmetry in the intramolecular charge distribution which cause an asymmetry in the repulsive potential also give rise to a quadrupole moment. In other words, $\psi$ and $Q$ have a common cause, but are not directly related. This conclusion must remain tentative, however. The derivation of Eq. (15) is very crude, and the dependence on distance is very strong. (If one assumes the zero energy collision diameter in place of the viscosity diameter, $\psi \sim 0.4$.)

\section{CONCLUSIONS}

Since the data on nitrogen, oxygen, carbon dioxide, methane, and hydrogen ${ }^{1}$ yield collision numbers for rotational relaxation which are generally in accord with values obtained by other experimental methods, we conclude that the approximate theory of Mason and Monchick ${ }^{5}$ is substantially correct.

Rotational relaxation times for near-spherical molecules such as methane, carbon tetrafluoride, sulfur hexafluoride, and even ethane are in good accord with theory for classical rough sphere molecules with attractive forces- $a$ reasonable model for these substances. Ethylene, which deviates markedly from spherical symmetry, has a smaller collision number-about half that calculated for the rough sphere molecule.

Collision numbers for linear molecules--nitrogen, oxygen, carbon dioxide, and acetylene--are in at least qualitative accord with an extension of Brout's ${ }^{11}$ quantum theory for rotational relaxation of homonuclear diatomic molecules. This theory indicates that the deviation of the repulsive force field of a molecule from spherical symmetry has an important effect.

In conclusion, it appears that the following factors are of profound importance in determining rotational relaxation times for non-polar molecules:

1. The mass distribution (characterized by the parameter $4 I / \mathrm{mo}^{2}$ ).

2 . The strength of the intermolecular attractive forces (characterized by $\epsilon / k T$ ).

3. The deviation of the molecular force field from spherical symmetry.

\section{ACKNOWLEDGMENTS}

We wish to thank Bonnie McBride and Janet Ehlers of the Physical Chemistry Branch, Lewis Research Center, for providing the ideal gas statistical mechanical entropy and heat capacity calculations for $\mathrm{CH}_{4}, \mathrm{CF}_{4}, \mathrm{SF}_{6}, \mathrm{C}_{2} \mathrm{H}_{4}, \mathrm{C}_{2} \mathrm{H}_{2}$, and $\mathrm{CO}_{2}$. 